\title{
Highly accurate local functional fingerprints and
}

\section{their stability}

Daouia I. Larabi ${ }^{1,2}$, Martin Gell ${ }^{1,2}$, Enrico Amico ${ }^{3,4}$, Simon B. Eickhoff ${ }^{1,2}$, Kaustubh R. Patil $^{1,2}$

${ }^{1}$ Institute of Neuroscience and Medicine, Brain \& Behaviour (INM-7), Research Centre Jülich, Jülich, Germany

${ }^{2}$ Institute of Systems Neuroscience, Medical Faculty, Heinrich Heine University Düsseldorf, Düsseldorf, Germany

${ }^{3}$ Institute of Bioengineering, Center for Neuroprosthetics, EPFL, Geneva, Switzerland ${ }^{4}$ Department of Radiology and Medical Informatics, University of Geneva, Switzerland 


\section{Abstract}

The neural underpinnings of individual identity reflected in cognition, behavior, and disease remain elusive. Functional connectivity profiles have been used as a "fingerprint" with which an individual can be identified in a dataset. These established functional connectivity fingerprints generally show high accuracy but are still sensitive to mental states. A truly unique, and especially state-independent, neural fingerprint will shed light on fundamental intra-individual brain organization. Moreover, a fingerprint that also captures inter-individual differences in brain-behavior associations will provide the necessary ingredients for the development of biomarkers for precision medicine. With resting-state and task fMRI-data of the Human Connectome Project and the enhanced Nathan Kline Institute sample, we show that the local functional fingerprint, and especially regional homogeneity (ReHo), is 1) a highly accurate neural fingerprint, 2) more stable within an individual regardless of their mental state (compared to the baseline functional connectome fingerprint), and 3) captures specific inter-individual differences. Our findings are replicable across parcellations as well as resilient to confounding effects. Further analyses showed that the attention networks and the Default Mode Network contributed most to individual "uniqueness". Moreover, with the OpenNeuro.ds000115 sample, we show that ReHo is also stable in individuals with schizophrenia and that its stability relates to intelligence subtest scores. Altogether, our findings show the potential of the application of local functional fingerprints in precision medicine. 


\section{Introduction}

Phenotypic traits exhibit population-level variability. Examples include physical and cognitive abilities, as well as brain organization. Identifying the inter-individual variability in functional brain organization that is predictive of cognitive and clinical properties at the individual level is important for developing viable biomarkers for precision medicine and precision psychiatry specifically (1). However, the highly dynamic and state-sensitive nature of brain function (2) makes it challenging to find trait-like functional properties of individual brains. Consequently, trait-like functional representations that are simultaneously stable within an individual and capture inter-individual differences are prerequisites for biomarker discovery that remain underdeveloped.

Whole-brain patterns of synchrony in fluctuations of the blood-oxygen-level-dependent (BOLD) signal as measured with functional magnetic resonance imaging (fMRI) - i.e. the functional connectome (FC) - is one of the most commonly used representations of brain function. The FC is usually calculated as the Pearson correlations between the time courses of all possible pairs of regions during resting-state or a task. The FC of an individual is highly stable and sufficiently unique to identify that individual across sessions or databases $(3,4)$, hence it has been termed a "neural fingerprint". This intra-individual stability satisfies one of the two critical requirements for potential clinical utility of an fMRI fingerprint $(5,6)$. The second critical consideration is whether a brain representation, such as the FC fingerprint, also captures inter-individual differences. The FC fingerprint has indeed been shown to capture individual differences (7). However, it has been argued that the two aspects - i.e. intra-individual stability and individual-level prediction of behavior - do not rely on the same information from the FC (8). Interestingly, the variation in the FC fingerprint measured across $\mathrm{FMRI}$ sessions, thus reflective of (task-related) reconfiguration, is lower in individuals with higher intelligence (9). Furthermore, the variability in the FC fingerprint has been associated with development, aging, and psychiatric disorders (10-14). Importantly, it can be indicative of mental health issues, e.g. schizophrenia, depression, or attention deficit disorder diagnoses (13). This provides a promising framework for satisfying the two prerequisites for biomarker discovery; representations of trait-like brain function whose reconfigurations, albeit subtle, could be predictive of inter-individual differences. Taken together, a trait-like state-independent neural fingerprint that captures inter-individual differences can shed light on fundamental brain organization as well as facilitate translational applications.

So far, the FC has been the choice to investigate neural fingerprints. However, the overall specificity of the FC fingerprint drastically drops when the mental state changes (15). For instance, in the seminal study of Finn et al. (2015), the high identification accuracy of $94.4 \%$ between two resting-state (rs) fMRI sessions dropped to $54 \%$ when comparing resting-state and task-based fMRI (3). This is not surprising as the FC is known to change according to situational demands (2), i.e. it captures state-dependent variance which is likely to differ between rest and task sessions. Here, we hypothesize that spatially restricted local brain measures, as opposed to global measures of synchrony between spatially distant regions, might be more state-independent and thus more stable within an individual. Good reproducibility (i.e. test-retest reliability) of local measures have been shown in rs-fMRI, while global measures performed poorly (16). So far, the prediction of subject identity has only 
been investigated with one local brain measure, namely region-specific variance in BOLD-activation (BOLD-variability; BV) (17). The use of other local measures for fingerprinting, such as regional homogeneity (ReHo), has not been investigated yet. Therefore, here, we investigate this hypothesis using three local fingerprints; ReHo, amplitude of low-frequency fluctuations (ALFF), and fractional ALFF (fALFF) within predefined brain regions based on the 264 Power coordinates (18). These local fingerprints were compared with the FC baseline. First, we quantified the intra-individual stability of these four fingerprints using the resting-state and task fMRI data of the Human Connectome Project. Importantly, in addition to using the conventional identification accuracies, we employed several methods to quantify the performance of each fingerprint, including identification in a forensic sense. So far identification studies have considered the most similar fingerprint as a match, in effect ignoring the possibility of a "no-match". We address this issue by defining a machine learning framework to predict whether two fingerprints match or not. We found near-perfect identification accuracies when using ReHo, followed by ALFF, fALFF, and the FC. Extensive evaluations showed replicability of our findings across parcellations and resilience against confounding effects. We then used a novel optimization formulation to investigate the regional specificity of the ReHo fingerprint. Next, we tested the intra-individual stability of fingerprints in a different clinical sample of individuals with schizophrenia, the OpenNeuro.ds000115 sample. Then we investigated the ability of fingerprint stability - i.e. invariance across mental states - to capture inter-individual variance by 1 ) predicting crystallized and fluid intelligence in a machine-learning setup in the large HCP sample, and 2) correlating stability with intelligence in individuals with schizophrenia and matched healthy controls of the OpenNeuro.ds000115 sample. Last, to address the question of neural origins of the BOLD signal (19), using data from the enhanced Nathan Kline Institute (eNKI) sample, we compared fingerprints between rs-fMRI and breath-holding scans that are known to capture cerebrovascular signals (20). Altogether, our findings suggest that measures of local neural activity, and especially ReHo, show high specificity, sensitivity, and robustness as a neural fingerprint.

\section{Results}

Analyses were performed using data of 399 unrelated subjects with two resting-state and seven task-based scans, acquired within 2 days, from the $\$ 1200$ release of the Human Connectome Project (HCP) (21). The brain was parcellated into 264 spherical regions with a 3-mm radius around the Power coordinates (18) (as analyses showed that smaller regions of interest (ROIs) yielded better identification accuracies - see Supplementary Fig. S1). The global FC fingerprint was calculated as the Pearson correlations between the mean time series of each pair of ROls (3). Furthermore, three local fingerprints were calculated: 1) ALFF: the averaged square root of the power in the $0.01-0.1 \mathrm{~Hz}$ frequency range averaged across voxels within a ROI (22)), 2) fALFF: ALFF scaled by the total power in the full frequency range averaged across voxels within a ROI (23)), and 3) ReHo: Kendall's coefficient of concordance considering the time series of all the voxels within an ROI (24). Thus, while the often-reported FC fingerprint reflects the similarity of the mean time series of each pair of (spatially distant) ROls, ReHo reflects the synchronization of the voxels' time series within a ROI, and (f)ALFF reflects BOLD-fluctuations within a ROI. Next, identification accuracies were calculated across all pairs of sessions. Iteratively, one subject's fingerprint 
was selected from one session ("base dataset" consisting of one fingerprint per subject) and Spearman correlated with all fingerprints of another session ("target dataset"). This yielded the similarity between the fingerprint of a subject in the "base dataset" with the fingerprints of all the subjects in the "target dataset". Using a 1-nearest neighbor approach (3), the "base" fingerprint was labeled with the subject identity of the most similar fingerprint in the "target" dataset. If the subject labeling was correct, this counted as an accurate identification. Identification accuracy was defined as the number of correctly labeled participants divided by the total number of participants. This was conducted in an iterative manner such that each session was used as the "base dataset" once. We observed near-perfect identification accuracies, i.e. high individual specificity when using ReHo, followed by ALFF, fALFF, and FC (Figure 1A). Next, we investigated whether our findings hold for several different ways of quantifying identification performance.

\section{Highly accurate individual identification based on local functional fingerprints irrespective of identification performance quantification}

Three additional ways to quantify identification performance were investigated. First, we used the often-reported "Idiff" score, which is the difference between the average within-subject and average between-subject similarity of a fingerprint; a higher Idiff indicates a better fingerprint (12). Idiff scores were consistent with the accuracy results; highest Idiff for ReHo, followed by ALFF, fALFF, and FC (Supplementary Fig. S2; for general interest additional results using a second parcellation (Dosenbach) are also reported). Second, we investigated the scenario in which there could be multiple fingerprints from the same subject in the target database. To this end, taking advantage of the multiple scans available in the HCP dataset, we defined a new score, called "ranking accuracy", to quantify how a fingerprint consistently ranks scans of different sessions of the same subject higher. This stricter method yielded the same pattern of results (Figure 1B). Note that at rank 2 the ranking accuracy is similar to the conventional identification accuracy where the FC fingerprint performs relatively similar to the local fingerprints but the difference between them becomes evident at higher ranks. Third, to simulate the scenario in which a subject's fingerprint might be missing in the database, we additionally calculated forensic identification. We used the two resting-state sessions as training data and induced a logistic regression model using a match-no-match dataset derived from $70 \%$ of the subjects. Each of the $30 \%$ held-out subjects was probed against all the subjects as a reference to obtain matches. The reference dataset was either the average of the two resting-state sessions or one of the task sessions (emulating the privacy-preserving scenario where the training data itself is not available). We tested two ways to derive features from the fingerprint pairs, (1) a single feature of correlation distance, and (2) absolute difference between the fingerprint elements. The results clearly demonstrate the superiority of the local fingerprints in forensic identification. The nearly zero true-positive and false-positive rates of FC indicate that it failed to match any of the test subjects (Fig. 1C). 
A
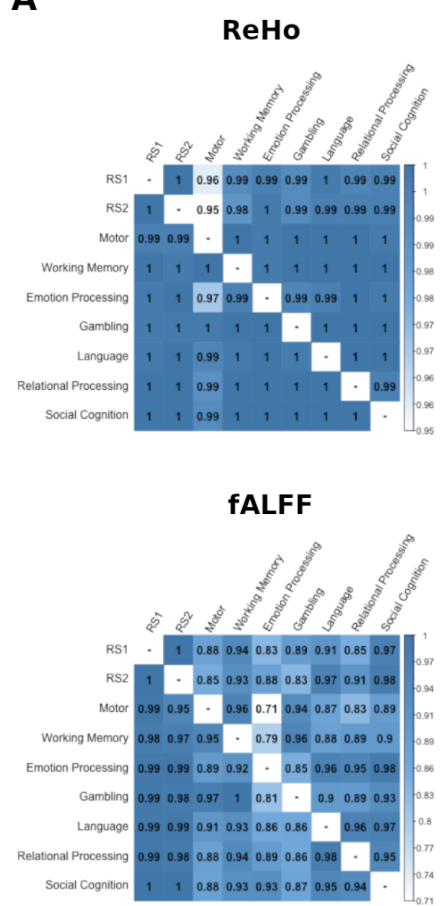

ALFF

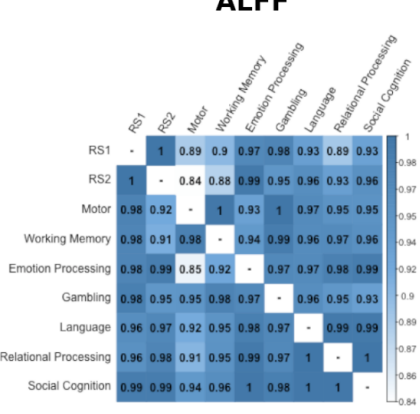

FC

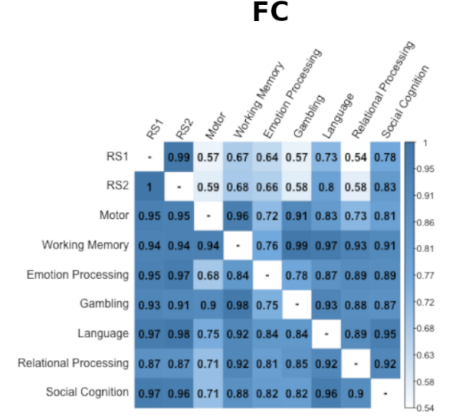

B

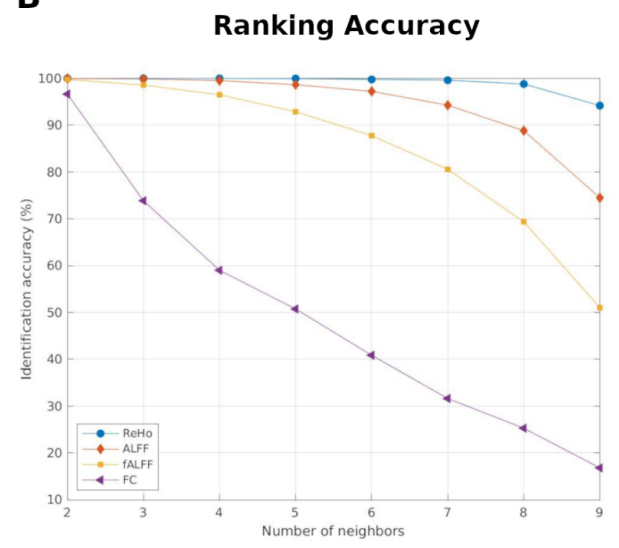

C Out-of-sample Prediction

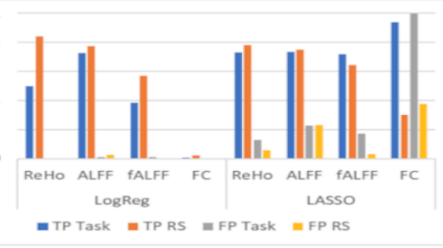

Figure 1: A) comparison of identification accuracies of functional fingerprints in the Human Connectome Project (HCP) sample; B) comparison of identification accuracies based on the ranking of functional fingerprints in the HCP sample; $\mathbf{C}$ ) forensic identification performance using fingerprint dissimilarity as a feature (LogReg) or the pattern of distances between the fingerprint elements as features (LASSO). Abbreviations: ReHo = regional homogeneity; ALFF = Amplitude of Low Frequency Fluctuations; fALFF = fractional Amplitude of Low Frequency Fluctuations; FC = functional connectome; RS = resting state; LogReg = logistic regression; $\mathrm{TP}=$ true positive rate; $\mathrm{FP}=$ false positive rate.

\section{Better identification performance of local functional fingerprints is replicable across parcellations and samples and resilient to confounding effects}

Also using the HCP dataset, we performed extensive evaluations to establish replicability and specificity of our results obtained with the Power $3 \mathrm{~mm}$ atlas. First, we tested three other parcellations, namely Dosenbach (25), Shen (26), and Schaefer with 300 ROIs (27), which also showed higher identification accuracies for the local fingerprints (Fig. S3). Similar to our results with the Power spheres, the Dosenbach parcellation showed the highest identification accuracies for ReHo, followed by ALFF, fALFF, and FC. With the Shen and Schaefer parcellations, results followed a similar pattern with a slight difference in the order: highest identification accuracies were found for ALFF, followed by ReHo, FC, and fALFF. Second, we investigated the effect of removing confounds, i.e. sex, age, and total intracranial volume, from the fingerprints. Removal of these confounds increased overall accuracy while retaining the result pattern - highest accuracy with ReHo (Fig. S4). Last, replication on the smaller Finn et al. (2015) HCP sample $(n=125)$ showed similar results with some minor differences (see Supplementary Materials for details; Fig. S5 \& S6). 


\section{Attention networks and the Default Mode Network contribute most to individual uniqueness}

Encouraged by these results, next, we investigated the contribution of each ROI in making the ReHo profile individual-specific. We posed this as an optimization problem using the weighted Pearson correlation coefficient to maximize the margin (i.e. the difference between the correct fingerprint and the next best) resulting in an importance weight for each ROI (see Supplementary Materials for details). We used the two resting-state sessions and the motor task HCP data with subjects included in Finn et al. (3). The optimization was performed 100 times with randomly selected $50 \%$ of the sample to obtain the weights that were then evaluated on the $50 \%$ hold-out sample. We observed that the margin increased substantially. The average ReHo across subjects showed an inverse $U$-shaped relationship with the weights (Fig. 2), indicating that ReHo values in the 0.2-0.25 range are most individual-specific. The proportion of the ROls in each of the seven Yeo networks (28) with their weight above a certain threshold indicated the highly weighted individual-specific ROls to be mostly located in dorsal- and ventral-attention networks followed by the default mode network, while the limbic network contained the fewest number of individual-specific ROls (Fig. 2; see details in Supplementary Materials).
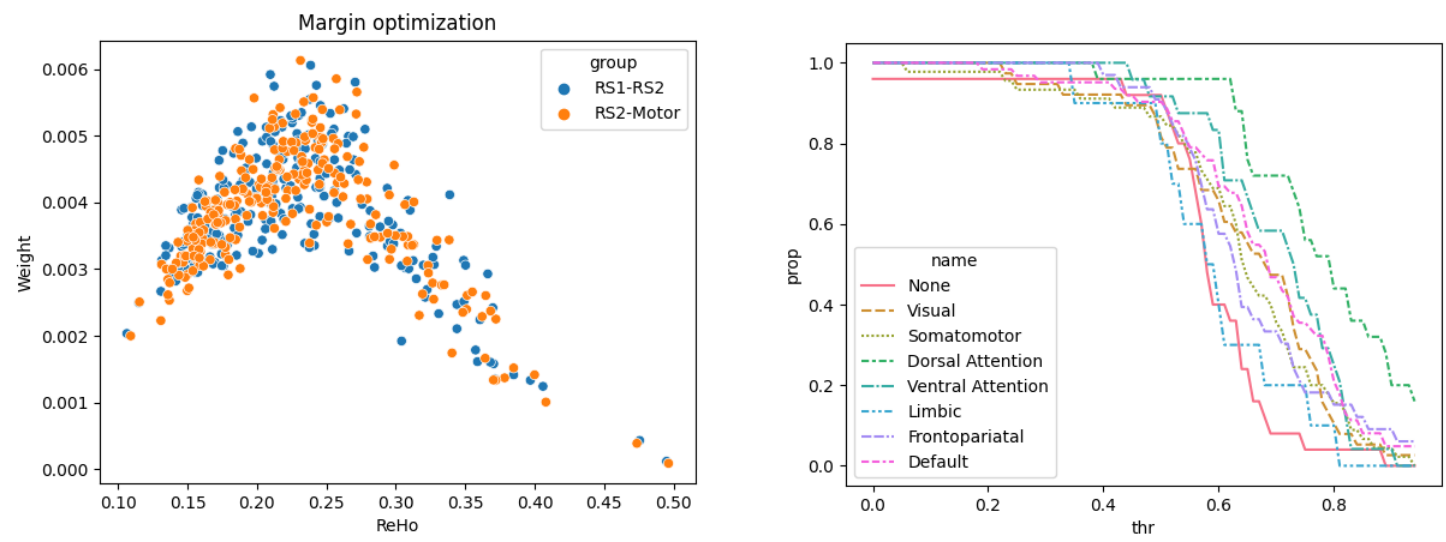

Figure 2: contribution of regions of interest (ROIs) in making ReHo individual-specific. Left) relationship between $\mathrm{ReHo}$ and averaged (across all optimization runs) importance weights of ROls. Right) proportion of ROls in Yeo's networks. Abbreviations: ReHo = regional homogeneity; RS1 = resting-state fMRI session day $1 ; \mathrm{RS} 2$ = resting-state $\mathrm{fMRI}$ session day 2; Motor $=\mathrm{fMRI}$ motor task; prop $=$ proportion; thr $=$ threshold .

\section{Local fingerprints are also stable within individuals of a clinical sample}

In addition to the healthy population, neural fingerprints also need to be stable within clinical samples for their use as clinical biomarkers. Therefore, next, we analyzed the OpenNeuro.ds000115 dataset (29) including a clinical sample diagnosed with schizophrenia $(S Z ; n=21)$ and healthy controls $(n=20$; see details in Supplementary Materials). fMRI data of the 0- and 2-back tasks, obtained the same day, were used. Delayed stabilization (14) and decreased stability of the FC fingerprint have been reported in schizophrenia (13). Studies 
have also shown local abnormalities in schizophrenia (30-32), but it is unclear whether these abnormalities are stable within patients. Identification accuracies for ReHo and ALFF were $100 \%$ for both controls and patients (analyzed separately) while they were lower in SZ for fALFF (65\% in SZ; $88 \%$ in HC) and FC (99\% in SZ; $100 \%$ in HC). This suggests that the ReHo and ALFF fingerprints are also stable in patients with schizophrenia.

\section{Fingerprint stability predicts intelligence}

Capturing inter-individual differences in behavior is a prerequisite of a biomarker. Therefore, next, we investigated the use of the stability of the fingerprints in the prediction of intelligence in the large HCP sample $(n=399)$. To investigate this, we first calculated - for each individual - the stability of the fingerprints as the average correlation of each session's fingerprint with the fingerprints of all other sessions resulting in a feature vector with nine values (i.e. two resting state- and seven task fMRI sessions). This feature space was then employed to predict individual-level crystallized and fluid intelligence in a 5 times repeated 5-fold cross-validation setup using least absolute shrinkage and selection operator (LASSO) regression (33). The variance due to age and total intracranial volume was removed from the features in a cross-validation consistent manner (34). We chose to predict intelligence as it is associated with task-related reconfiguration of brain networks, i.e. lower reconfiguration is associated with higher intelligence (9). We observed that the stability of ReHo was highly predictive of crystallized intelligence, ALFF stability predicted both crystallized and fluid intelligence, while the stability of fALFF and FC was generally not predictive (Fig. 3A). A closer inspection of the LASSO weights for the ReHo features for predicting crystallized intelligence revealed that higher ReHo stability between resting state and the other sessions is predictive of higher crystallized intelligence, while higher ReHo stability between the language task and the other sessions is predictive of lower crystallized intelligence. In addition, for the prediction of crystallized intelligence, high positive coefficients were observed for ALFF stability of the motor, language, and gambling tasks. For fluid intelligence, the motor and relational processing task's ALFF stability received a high positive coefficient, and the social cognition task stability a negative coefficient.

Next, we also investigated the association between the stability of the fingerprints and intelligence in the schizophrenia sample and their matched healthy controls. Using the OpenNeuro.ds 000115 dataset, we correlated the stability of the fingerprints with the Wechsler Adult Intelligence Scale (WAIS) Matrix Reasoning and Vocabulary subtest scores in both the $\mathrm{SZ}$ and $\mathrm{HC}$ groups. Stability was calculated as the correlation between the fingerprints of the 0-back and 2-back task sessions. Machine learning analysis was not attempted here due to the small sample size. In the SZ group, stability of the ReHo, ALFF, and FC fingerprints showed a high negative correlation with the WAIS Matrix Reasoning subtest (all $r<-0.50$, Fig. 3B), while in the HC group only ALFF stability showed a positive correlation with WAIS Vocabulary subtest scores $(r=0.48)$. 

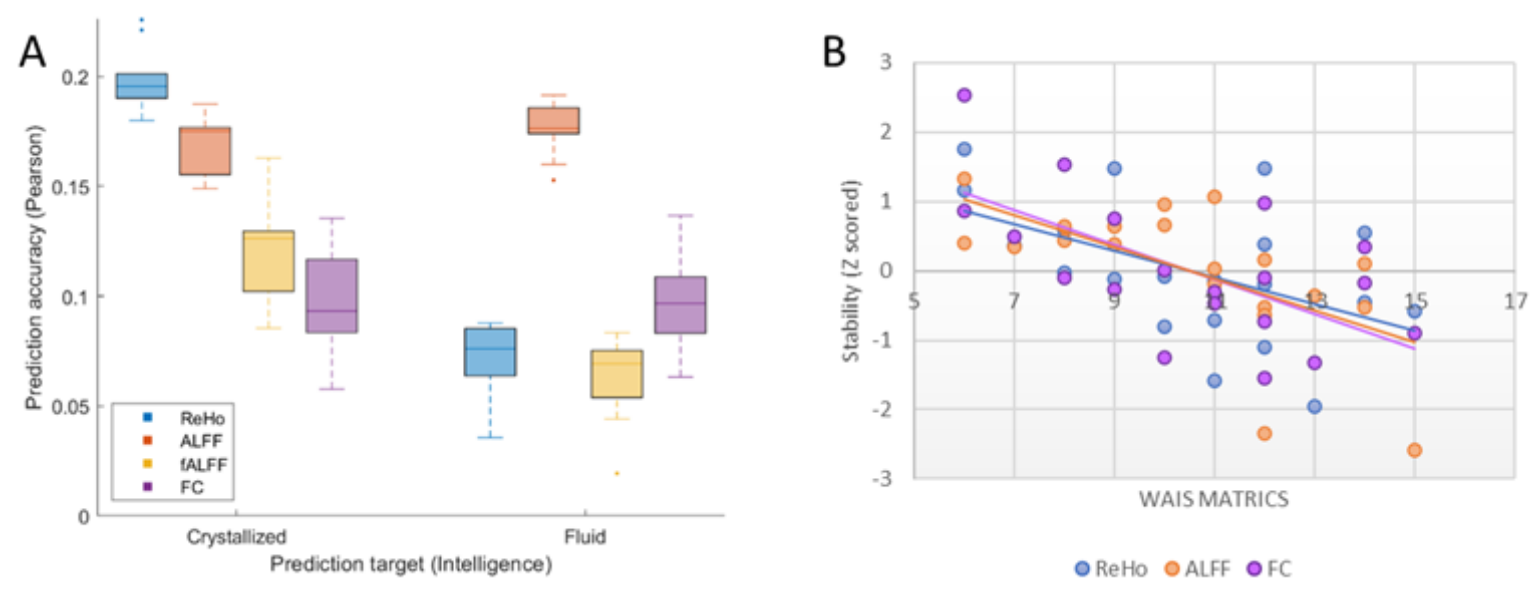

Figure 3: A) Prediction of crystallized and fluid intelligence using the stability of fingerprints in the nine sessions of the Human Connectome Project sample. Each boxplot shows the prediction performance across five cross-validation runs; B) scatterplot between the WAIS Matrix Reasoning scores and the stability of the task fingerprints in the SZ sample of the OpenNeuro.ds000115 dataset. Abbreviations: ReHo = regional homogeneity; ALFF = Amplitude of Low Frequency Fluctuations; fALFF = fractional Amplitude of Low Frequency Fluctuations; $F C$ = functional connectome.

\section{ReHo represents a neural fingerprint}

Altogether, thus far, our findings suggest that ReHo is most stable within an individual and also captures brain-behavior associations. However, its high individual-specificity might be driven by stable non-neural signals (i.e. artifacts, breathing, cardiovascular effects). Indeed, earlier studies have shown high stability of motion and cardiac pulsatility, and that test-retest reliability reduces after artifact removal (10). Therefore, as a follow-up analysis, we investigated the contribution of vascular activity to local and global fingerprints. Previous research showed that breath-holding is a reliable estimate of the influence of vascular activity on BOLD signal (35) as it induces hypercapnia: an increase in carbon dioxide causing increased cerebral blood flow. This was investigated with the resting-state and breath-holding fMRI sessions (TR of $1400 \mathrm{~ms}$ ) provided in the enhanced Nathan Kline Institute dataset (eNKI; n=478; see Supplementary Materials for details) (36), as the HCP does not provide breath-holding fMRI data. The breath-holding paradigm consists of alternating breathing in, out, and breath-holding, causing a global change in oxygenation. We observed the highest identification accuracies between resting-state and breath-holding with ALFF (99\%), followed by ReHo (87\%), FC (72\%), and fALFF (44\%) (Fig. S9 \& S10). This suggests that the vascular signal in resting-state $\mathrm{fMRI}$ is captured with all fingerprints, but especially with ALFF.

\section{Discussion}

Using four different ways for the quantification of identification performance, different parcellations, and ROI sizes, our findings show highly accurate local functional fingerprints, especially when using ReHo. 


\section{Regional homogeneity is a highly accurate local fingerprint irrespective of parcellation choice, sample size, and confounding effects}

In addition to the results obtained with ROls created with 3-mm spheres around the Power coordinates, identification was also evaluated using three other parcellations (i.e.

Dosenbach, Shen, and Schaefer with $300 \mathrm{ROIs})$. While results with the Dosenbach parcellation were the same, for the Shen and Schaefer parcellations, higher identification accuracies were found for ALFF, followed by ReHo, FC, and fALFF (versus ReHo, ALFF, fALFF, FC with Power and Dosenbach parcellations). This difference might be explained by the fact that ROls in the Power (264 ROIs; 3-mm radius) and Dosenbach (160 ROls; 5-mm radius) parcellations are compact spheres while the parcels of the Shen (268 ROIs) and Schaefer (300 ROIs) are much larger. This explanation also holds for our finding that higher identification accuracies were found for fALFF with the Power and Dosenbach parcellations, while the FC performed better with the Shen and Schaefer parcellations. These larger ROIs reflect more macroscopic organization, are impacted more by partial volume effects, and averaging within these larger ROls affects local fingerprints more. As we calculated ReHo for each ROI as Kendall's coefficient of concordance for all the voxel-wise time series within that $\mathrm{ROI}, \mathrm{ReHo}$ values are expected to converge to 0 with increasing parcel size, in effect rendering the ReHo-fingerprint less specific and confirming the importance of spatial resolution in ReHo calculation. Second, our finding of highly accurate local fingerprints was resilient against confounding effects, as confound removal resulted in the same pattern of identification accuracies, with ReHo yielding highest accuracies. Confound removal benefited identification accuracies for FC the most and ALFF the least, suggesting higher sensitivity of the FC and lower sensitivity of ALFF to confounding effects. Third, it is expected that larger samples yield lower identification accuracies. Therefore, we first replicated the results reported in Finn et al. (2015). Next, with the same sample of only 125 subjects, we also compared identification accuracies of the global fingerprint to those of local fingerprints. We found that identification accuracies for the FC reduced most with larger sample sizes, while local fingerprints, and especially ReHo, were affected the least. For the Power and Dosenbach parcellations, our investigation on the smaller sample of 125 subjects showed one difference compared to results in the larger HCP sample: identification accuracy was higher for the FC than for fALFF. Altogether, our findings suggest that ReHo shows high specificity, sensitivity, and robustness as a neural fingerprint.

\section{Attention networks and the DMN contribute most to individual "uniqueness"}

Weighting ReHo features higher in some brain regions enhanced the fingerprint efficacy, revealing brain regions that contribute more to individual uniqueness. We found that regions in the dorsal- and ventral-attention networks and the default mode network contributed most to identifiability, while the limbic network contributed the least. This is in line with previous studies showing that higher-order cognitive systems, or frontoparietal regions, contribute to identifiability, in contrast to limbic regions (37). However, it should be noted that limbic regions are smaller than ROls within the other networks, and therefore might be affected more by normalization errors that induce noise. 


\section{The stability of fingerprints captures inter-individual differences}

Next, we predicted intelligence with the stability of the fingerprints across sessions. Our results showed that the stability of ReHo could predict crystallized intelligence but not fluid intelligence. On the other hand, ALFF stability could predict both crystallized and fluid intelligence, while fALFF and the FC were not predictive. This discrepancy suggests that different fingerprints and their stability or reconfiguration will be more appropriate for predicting different targets.

Regional homogeneity $(\mathrm{ReHo})$ is calculated as the synchronization of the time series of voxels within a ROI. The stability of ReHo across states appears to reflect the existence of regions that work relatively uniformly across mental states. Our findings suggest this might be associated more with the more static form of intelligence that is conceptualized with crystallized intelligence, than with the more dynamic ability to reason and solve new problems referred to as fluid intelligence - which might be associated more with brain dynamics. A closer inspection of the LASSO weights for the ReHo features for predicting crystallized intelligence revealed that higher ReHo stability between resting state and the other sessions is predictive of higher crystallized intelligence, while higher ReHo stability between the language task and the other sessions is predictive of lower crystallized intelligence. These observations suggest that reconfigurations of ReHo across states are smaller in individuals with higher crystallized intelligence, but reconfigurations during a language task are larger. This is in line with a study showing that the FC at rest was more similar to the FC during several tasks in individuals with higher intelligence. This ability to reconfigure networks efficiently based on task demands can be seen as a sign of high intelligence (9).

Furthermore, for individuals with schizophrenia of the OpenNeuro.ds000115 dataset, the higher stability of ReHo, ALFF, and FC fingerprints was associated with lower scores on the Matrix Reasoning subtest. This subtest measures abstract problem solving and inductive reasoning, which is considered part of fluid intelligence. Furthermore, the 0-back task requires sustained attention, while the 2-back task additionally requires working memory. Thus, ReHo, ALFF, and FC fingerprints are more stable between these two tasks in individuals with schizophrenia and lower fluid intelligence, confirming differing demands of these two tasks. However, this was not found in the matched $\mathrm{HC}$ of the OpenNeuro.ds000115 dataset (all $r<0.02-0.1$ ). In HC, only ALFF stability was associated with scores on the Vocabulary subtest. Altogether, our findings suggest that even though the ReHo and ALFF fingerprints are highly stable (Fig. 1), subtle variation due to mental states is still informative.

\section{ALFF is affected more by vascular signals than ReHo}

Identification accuracies with the eNKI breath-holding fMRI-task and resting-state data suggest that breathing, or vascular signal, is represented more in resting-state ALFF than in resting-state ReHo. Indeed, previous research has shown that ALFF captures this signal (38). Furthermore, our results are in line with the idea that fALFF, as a normalized ALFF, is affected less by physiological signals from large blood vessels and CSF that affect the entire frequency spectrum. Taken together, the high accuracy of ReHo in the HCP samples and its 
lower accuracy with breath-holding data suggests that ReHo captures neural signals more than ALFF.

\section{Limitations}

This study has some limitations. First, the contribution of physiological signals to the BOLD signal is unclear. We tested the contribution of vascular activity to the fingerprints, but other sources of physiological signal might also contribute. Second, we created fingerprints based on local brain function, while other studies have investigated fingerprints based on shape, white matter fiber geometry, voxel-wise diffusion density, or combinations (39). Future studies could investigate a multimodal fingerprint including ReHo. Third, we analyzed data that was acquired within a short timespan (i.e. days), but the stability of fingerprints over longer periods is unclear. Future work could examine the stability of local and global fingerprints over months or years. Fourth, we checked fingerprint stability in one, relatively small, clinical sample of individuals with schizophrenia. It is unclear whether our findings generalize to other clinical samples.

\section{Conclusion}

Investigating associations between brain function and cognition, behavior, or disease characteristics (e.g. progression or severity) requires within-individual stability (40).

Altogether, our results suggest that measures of local neural activity are more stable fingerprints - i.e. more stable within an individual regardless of their mental state. This was replicable across parcellations as well as resilient to confounding effects. The near-perfect identification of the ReHo fingerprint between rest- and task-fMRI combined with its reduced accuracy with breath-holding data suggests that it largely reflects neuronal signal and that it can be indeed deemed a "neural fingerprint" - making it especially attractive for future studies of brain-behavior relationship and clinical applications. As ReHo reflects the synchronized activation of voxels within a parcel, the intraindividual stability of ReHo supports the existence of regions that work uniformly across mental states. The FC, on the contrary, captures dynamic and state-dependent interactions between regions and might be less suitable for answering identifiability questions. While studies have shown ReHo abnormalities across disorders such as autism (30) and schizophrenia (31), our findings suggest that ReHo measures in schizophrenia are stable within an individual. Furthermore, the use of weighted ROIs increases individuality and therefore identifiability. When interpreted with recent findings (8), neural representations capturing both intra-individual stability and inter-individual differences (both desired properties for clinical biomarkers), remain enigmatic. Altogether, accurate fingerprints - such as the one we propose here based on ReHo - provide an individual-specific neural signal and can help in this endeavor, benefiting both basic and clinical investigations. 


\section{Methods and Materials}

\section{Calculation of identification accuracy in Human Connectome Project sample}

\section{Human Connectome Project (HCP) sample}

Data collection was approved by the Institutional Review Board at Washington University in St. Louis. The selection of subjects was done in R version 3.6.1 (R Core Team 2019). Individuals were included in case of availability of 1) a T1-weighted scan, 2) at least two resting-state fMRI scans (rfMRI_REST1 and rfMRI_REST2), 3) data on 58 behavioral variables (41) and 4) all fMRI-tasks (working memory (tfMRI_WM), gambling (tfMRI_GAMBLING), motor (tfMRI_MOTOR), language (tfMRI_LANGUAGE), social cognition (tfMRI_SOCIAL), relational processing (tfMRI_RELATIONAL) and emotion processing (tfMRI_EMOTION) (42). Subjects with reported issues according to the HCP data release updates were excluded. From this sample, subjects were selected that were unrelated (variable Family_ID) with a similar number of selected males and females (Gender) that were matched on age (Age_in_Yrs), education (SSAGA_Educ), race (Race), and BMI (BMI). This resulted in a sample of 399 subjects consisting of 195 females and 204 males. The mean age, education, and BMI were similar between males and females,

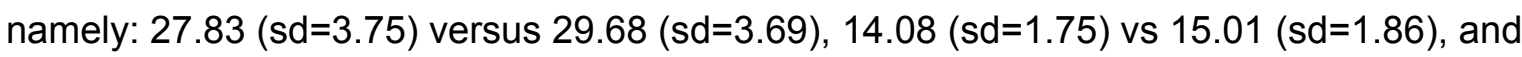

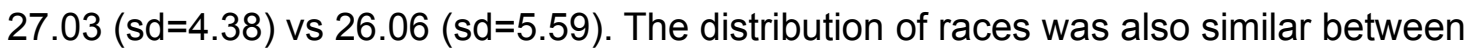
males and females: Asian/Nat. Hawaiian/Other Pacific Is. (11 vs 13), Black or African Am. (28 vs 29 ), White (154 vs 145), unknown or not reported (4 vs 2), more than one (7 vs 6 ). For all subjects, all available fMRI data (i.e. two resting-state fMRI-sessions and seven fMRI-tasks) were used for the main analyses. Further details on the tasks and acquisition parameters can be found in Barch et al., 2013 (42).

We used the preprocessed data provided by the HCP, which includes artifact removal, motion correction, and registration to standard space. For the resting-state data, the ICA-FIX noise components removed data were used. We also used the sample from Finn et al. (3) $(n=125)$ for replication and optimization analyses.

\section{Preprocessing}

Additional minimal preprocessing was done assuming no prior knowledge of the data, therefore allowing the use of our preprocessing pipeline in a broad range of situations (i.e. task- or resting-state data, variable scan parameters, etc.). This was done in MATLAB release 2019b (MathWorks) and included: regressing out the 12 detrended motion parameters (provided by HCP as Movement_Regressors_dt.txt), as well as mean time courses of WM, CSF, and global signal, and filtering the time series with a bandpass filter of 0.01-0.1 Hz. Next, the fingerprints were calculated for both left-right (LR) and right-left (RL) phase-encoding runs and consequently averaged. 


\section{Local fingerprints}

A local fingerprint for a subject consists of a vector of length $\mathrm{N}$ where $\mathrm{N}$ is the number of ROls.

\section{Global fingerprint (whole-brain functional connectome)}

A FC for a subject consists of a matrix of size $\mathrm{NxN}$ where $\mathrm{N}$ is the number of ROls. We extracted the lower triangle of this matrix and vectorized it to get the $\mathrm{FC}$ fingerprint as a vector of length $\mathrm{N} \times(\mathrm{N}-1) / 2$.

\section{Idiff}

The within-subject similarity of FCs ("Iself") is the average of main diagonal elements of the identification matrix, while the between-subjects similarity of FCs ("lothers") is the average of the off-diagonal elements. Idiff is then calculated by taking the difference between Iself and lothers and multiplying it with 100 (12).

\section{Ranking accuracy}

All subjects in the target datasets are ordered based on their similarity with the subject's fingerprint in the base dataset, and the number of correctly ranked subjects is counted for each consecutive rank.

\section{Forensic identification}

The two resting-state sessions were used for training. First, we derived a match-no-match dataset. The match part of this dataset contained the features derived from fingerprints of the same subjects from the two sessions. The no-match data consisted of the features derived using each subject in session 1 and their other closest match from session 2 . We tested two feature spaces, (1) a single feature as the correlation distance between a fingerprint pair, and (2) absolute difference between the fingerprint elements providing a feature space of the same size as the fingerprint. This provided a dataset with $2 \times \mathrm{N}$ rows $(\mathrm{N}=$ number of subjects) which together with class labels indicating match or no-match was used to train a classifier. More specifically, we employed logistic regression with feature space 1 and LASSO with feature space 2. Given a reference fingerprint (with known identity) and a test fingerprint (unknown identity), a trained model was used to predict whether they match or not. A prediction was then obtained using each fingerprint in a reference database. Note that predictions for a test case can contain one, multiple, or no matches. If the correct subject was predicted as a match then it was counted as a true positive and if any other subject was predicted as a match then this was counted as a false positive. A good classifier should provide a high true-positive rate and a low false-positive rate. We randomly chose $70 \%$ of the subjects for training and used the rest for testing. This split was repeated 10 times. Ten times repeated 10 -fold cross-validation was performed on the training data to estimate the generalization ability. We tested two kinds of reference databases, the training fingerprints (averaged across both sessions) or fingerprints from another session to emulate the privacy-preserving scenario where only the classifier can be shared but not the training data. 


\section{Confound removal}

Confounding signals due to age, sex, and total intracranial volume (ICV) were regressed out from each column of each database (subjects $\mathrm{x}$ fingerprint) and the residuals were used for identification.

\section{References}

1. B. S. Fernandes, et al., The new field of "precision psychiatry". BMC Med. 15, 80 (2017).

2. M. G. Preti, T. A. Bolton, D. Van De Ville, The dynamic functional connectome: State-of-the-art and perspectives. Neuroimage 160, 41-54 (2017).

3. E. S. Finn, et al., Functional connectome fingerprinting: identifying individuals using patterns of brain connectivity. Nat. Neurosci. 18, 1664-1671 (2015).

4. B. A. Seitzman, et al., Trait-like variants in human functional brain networks. Proc Natl Acad Sci USA 116, 22851-22861 (2019).

5. M. P. Milham, J. Vogelstein, T. Xu, Removing the reliability bottleneck in functional magnetic resonance imaging research to achieve clinical utility. JAMA Psychiatry (2021) https:/doi.org/10.1001/jamapsychiatry.2020.4272.

6. P. Sorrentino, et al., Clinical connectome fingerprints of cognitive decline. BioRxiv (2020) https:/doi.org/10.1101/2020.10.09.332635.

7. E. S. Finn, M. D. Rosenberg, Beyond fingerprinting: Choosing predictive connectomes over reliable connectomes. Neuroimage 239, 118254 (2021).

8. M. Mantwill, M. Gell, S. Krohn, C. Finke, Fingerprinting and behavioural prediction rest on distinct functional systems of the human connectome. BioRxiv (2021) https:/doi.org/10.1101/2021.02.07.429922.

9. D. H. Schultz, M. W. Cole, Higher Intelligence Is Associated with Less Task-Related Brain Network Reconfiguration. J. Neurosci. 36, 8551-8561 (2016).

10. S. Noble, D. Scheinost, R. T. Constable, A decade of test-retest reliability of functional connectivity: A systematic review and meta-analysis. Neuroimage 203, 116157 (2019).

11. O. Miranda-Dominguez, et al., Connectotyping: model based fingerprinting of the functional connectome. PLOS ONE 9, e111048 (2014).

12. E. Amico, J. Goñi, The quest for identifiability in human functional connectomes. Sci. Rep. 8, 8254 (2018).

13. T. Kaufmann, et al., Stability of the brain functional connectome fingerprint in individuals with schizophrenia. JAMA Psychiatry 75, 749-751 (2018).

14. T. Kaufmann, et al., Delayed stabilization and individualization in connectome development are related to psychiatric disorders. Nat. Neurosci. 20, 513-515 (2017).

15. E. S. Finn, et al., Can brain state be manipulated to emphasize individual differences 
in functional connectivity? Neuroimage 160, 140-151 (2017).

16. Š. Holiga, et al., Test-retest reliability of task-based and resting-state blood oxygen level dependence and cerebral blood flow measures. PLoS ONE 13, e0206583 (2018).

17. G. Gaut, et al., Predicting Task and Subject Differences with Functional Connectivity and Blood-Oxygen-Level-Dependent Variability. Brain Connect. 9, 451-463 (2019).

18. J. D. Power, et al., Functional network organization of the human brain. Neuron $\mathbf{7 2}$, 665-678 (2011).

19. O. J. Arthurs, S. Boniface, How well do we understand the neural origins of the fMRI BOLD signal? Trends Neurosci. 25, 27-31 (2002).

20. J. Pinto, M. G. Bright, D. P. Bulte, P. Figueiredo, Cerebrovascular reactivity mapping without gas challenges: A methodological guide. Front. Physiol. 11, 608475 (2020).

21. D. C. Van Essen, et al., The WU-Minn Human Connectome Project: an overview. Neuroimage 80, 62-79 (2013).

22. Y.-F. Zang, et al., Altered baseline brain activity in children with ADHD revealed by resting-state functional MRI. Brain Dev. 29, 83-91 (2007).

23. Q.-H. Zou, et al., An improved approach to detection of amplitude of low-frequency fluctuation (ALFF) for resting-state fMRI: fractional ALFF. J. Neurosci. Methods 172, 137-141 (2008).

24. Y. Zang, T. Jiang, Y. Lu, Y. He, L. Tian, Regional homogeneity approach to fMRI data analysis. Neuroimage 22, 394-400 (2004).

25. N. U. F. Dosenbach, et al., Prediction of individual brain maturity using fMRI. Science 329, 1358-1361 (2010).

26. X. Shen, F. Tokoglu, X. Papademetris, R. T. Constable, Groupwise whole-brain parcellation from resting-state fMRI data for network node identification. Neuroimage 82, 403-415 (2013).

27. A. Schaefer, et al., Local-Global Parcellation of the Human Cerebral Cortex from Intrinsic Functional Connectivity MRI. Cereb. Cortex 28, 3095-3114 (2018).

28. B. T. T. Yeo, et al., The organization of the human cerebral cortex estimated by intrinsic functional connectivity. J. Neurophysiol. 106, 1125-1165 (2011).

29. G. Repovš, D. M. Barch, Working memory related brain network connectivity in individuals with schizophrenia and their siblings. Front. Hum. Neurosci. 6, 137 (2012).

30. D. K. Shukla, B. Keehn, R. A. Müller, Regional homogeneity of fMRI time series in autism spectrum disorders. Neurosci. Lett. 476, 46-51 (2010).

31. X. Zhao, et al., Abnormalities of regional homogeneity and its correlation with clinical symptoms in Naïve patients with first-episode schizophrenia. Brain Imaging Behav. 13, 503-513 (2019).

32. P. Wang, et al., Amplitude of low-frequency fluctuation (ALFF) may be associated with cognitive impairment in schizophrenia: a correlation study. BMC Psychiatry 19, 30 
(2019).

33. T. Hastie, R. Tibshirani, J. Friedman, The Elements of Statistical Learning, second (2009).

34. S. More, S. B. Eickhoff, J. Caspers, K. R. Patil, "Confound removal and normalization in practice: A neuroimaging based sex prediction case study" in Machine Learning and Knowledge Discovery in Databases. Applied Data Science and Demo Track: European Conference, ECML PKDD 2020, Ghent, Belgium, September 14-18, 2020, Proceedings, Part V, Lecture notes in computer science., Y. Dong, G. Ifrim, D. Mladenić, C. Saunders, S. Van Hoecke, Eds. (Springer International Publishing, 2021), pp. 3-18.

35. R. Yuan, et al., Regional homogeneity of resting-state fMRI contributes to both neurovascular and task activation variations. Magn. Reson. Imaging 31, 1492-1500 (2013).

36. K. B. Nooner, et al., The NKI-Rockland Sample: A Model for Accelerating the Pace of Discovery Science in Psychiatry. Front. Neurosci. 6, 152 (2012).

37. Y. Jo, J. Faskowitz, F. Z. Esfahlani, O. Sporns, R. F. Betzel, Subject identification using edge-centric functional connectivity. BioRxiv (2020) https:/doi.org/10.1101/2020.09.13.291898.

38. A. M. Golestani, L. L. Wei, J. J. Chen, Quantitative mapping of cerebrovascular reactivity using resting-state BOLD fMRI: Validation in healthy adults. Neuroimage 138 , 147-163 (2016).

39. K. Kumar, M. Toews, L. Chauvin, O. Colliot, C. Desrosiers, Multi-modal brain fingerprinting: A manifold approximation based framework. Neuroimage 183, 212-226 (2018).

40. U. Tipnis, et al., Data Release and Assessment on Brain Fingerprints (2020).

41. R. Kong, et al., Spatial Topography of Individual-Specific Cortical Networks Predicts Human Cognition, Personality, and Emotion. Cereb. Cortex 29, 2533-2551 (2019).

42. D. M. Barch, et al., Function in the human connectome: task-fMRI and individual differences in behavior. Neuroimage 80, 169-189 (2013).

\section{Acknowledgments and funding sources}

This work was supported by The Helmholtz Portfolio Theme 'Supercomputing and Modelling for the Human Brain' and the European Union's Horizon 2020 Research and Innovation Programme (HBP SGA2; Grant No. 785907 to SBE). The authors would like to thank Dr. Branislava Ćurčić-Blake for helpful discussions and Dr. Emily S. Finn for valuable comments on a previous version of this manuscript. 